\title{
Nonperturbative Determination of Heavy Meson Bound States
}

\author{
Chi-Yee Cheung ${ }^{a}$ and Wei-Min Zhang ${ }^{b}$ \\ ${ }^{a}$ Institute of Physics, Academia Sinica, Taipei, Taiwan 115, R.O.C. \\ ${ }^{b}$ Department of Physics, National Cheng-Kung University, Tainan, Taiwan 701, R.O.C.
}

(Dec. 20, 1998)

\begin{abstract}
In this paper we obtain a heavy meson bound state equation from the heavy quark equation of motion in heavy quark effective theory (HQET) and the heavy meson effective field theory we developed very recently. The bound state equation is a covariant extention of the light-front bound state equation for heavy mesons derived from light-front QCD and HQET. We determine the covariant heavy meson wave function variationally by minimizing the binding energy $\bar{\Lambda}$. Subsequently the other basic HQET parameters $\lambda_{1}$ and $\lambda_{2}$, and the heavy quark masses $m_{b}$ and $m_{c}$ can also be consistently determined.
\end{abstract}

PACS numbers: 12.38.-t, 12.39.Hg, 12.39.Ki, 12.60.Rc, 14.40.-n

Typeset using REVTEX 


\section{INTRODUCTION}

Very recently, we have developed an effective field theory to describe the nonperturbative QCD dynamics of heavy mesons [1,2]. This theory has incorporated in it heavy quark effective theory (HQET), and is thus consistent with heavy quark symmetry (HQS) in the infinite quark mass $\left(m_{Q} \rightarrow \infty\right)$ limit. In this effective field theory, a heavy meson is considered as a composite particle consisting of a reduced heavy quark (heavy quark in the $m_{Q} \rightarrow \infty$ limit) coupled with the light degrees of freedom. A structure function $\Psi\left(v \cdot p_{q}\right)$, corresponding to the wave function of the heavy meson bound state, is introduced to describe the quark-meson vertex in the effective Lagrangian. By combining such an effective Lagrangian for the composite heavy mesons with the $1 / m_{Q}$ expansion of the heavy quark QCD Lagrangian, we can systematically evaluate various $1 / m_{Q}$ corrections to heavy meson properties in a covariant field-theoretic framework [2].

In our previous works [1, [2], the heavy meson wave function $\Psi\left(v \cdot p_{q}\right)$ is taken to be a phenomenological function. Specifically it is assumed to be a Lorentzian function, $\Psi\left(v \cdot p_{q}\right)=$ $1 /\left(v \cdot p_{q}+\omega\right)^{n}$, in which the parameters $\omega$ and $n$ are determined phenomenologically by fitting to the $B$ meson decay constant $f_{B}$; hence its relation to nonperturbative low-energy QCD dynamics is not clear. In this paper, we attempt to determine the heavy meson wave function from a QCD-based bound state equation. The bound state equation of heavy mesons is obtain from the heavy quark equation of motion in HQET which is incorporated in our effective field theory. This heavy meson bound state equation is a covariant extension of the light-front bound state equation we obtained recently from light-front QCD and HQET [3]. With the bound state equation for $\Psi\left(v \cdot p_{q}\right)$, we can use a variational procedure to determine its soluton.

The paper is organized as follows: In Sec. II, we derive from the heavy quark equation of motion in the heavy quark limit and our effective heavy meson field theory the heavy meson bound state equation. Then a variational method is used to determine the heavy meson wave function $\Psi\left(v \cdot p_{q}\right)$ by minimizing the reduced mass (binding energy) of the heavy meson $\bar{\Lambda}$. 
In the literature, $\bar{\Lambda}$ has only been estimated either from heavy meson mass spectrum or from inclusive heavy meson decay data 四. A direct calculation of $\bar{\Lambda}$ has not been carried out to date. In Sec. III, we consistently calculate the other two basic HQET parameters $\lambda_{1}$ and $\lambda_{2}$ directly from the operators $\mathcal{O}_{1}$ and $\mathcal{O}_{2}$ in HQET. Having self-consistently calculated $\lambda_{1}$ and $\lambda_{2}$, we can then extract from experimental heavy meson masses the heavy quark masses $\left(m_{b}, m_{c}\right)$ at the low energy scale where our effective field theory applies. Such a procedure establishes a link between our heavy meson effective field theory and nonperturbative QCD dynamics. Finally a summary is given in Sec. IV.

\section{HEAVY MESON BOUND STATE EQUATION IN EFFECTIVE FIELD THEORY}

\section{A. Heavy meson effective field theory}

Before deriving the heavy meson bound state equation, we shall briefly review the heavy meson effecive field theory we developed recently [1,2]. In heavy quark effective theory (HQET) [5], the QCD Lagrangian is expanded in powers of $1 / m_{Q}$ :

$$
\begin{aligned}
\mathcal{L}_{Q C D}= & \bar{h}_{v} i v \cdot D h_{v}+\bar{q}\left(i \not D-m_{q}\right) q-\frac{1}{4} F_{a}^{\mu \nu} F_{a \mu \nu} \\
& +\sum_{n=1}^{\infty}\left(\frac{1}{2 m_{Q}}\right)^{n} \bar{h}_{v}\left(i \not D_{\perp}\right)(-i v \cdot D)^{n-1}\left(i \not D_{\perp}\right) h_{v} \\
= & \mathcal{L}_{0}+\mathcal{L}_{m_{Q}}
\end{aligned}
$$

where the reduced heavy quark field $h_{v}$ is defined from the full heavy quark field $Q$ by

$$
Q(x)=e^{-i m_{Q} v \cdot x} h_{v}+O\left(1 / m_{Q}\right), \quad \frac{1+\not p}{2} h_{v}=h_{v},
$$

$v^{\mu}$ is the heavy quark four velocity which is defined to be equal to that of the parent heavy meson, and $D_{\perp}^{\mu} \equiv D^{\mu}-v^{\mu} v \cdot D$. It is easily seen that the leading term $\mathcal{L}_{0}=\bar{h}_{v} i v \cdot D h_{v}$ in Eq.(2.1) possesses a $S U_{f}\left(N_{f}\right) \otimes S U_{s}(2)$ flavor-spin symmetry (or simply heavy quark

symmetry, HQS), whereas the symmetry breaking part $\mathcal{L}_{m_{Q}}$ contains $1 / m_{Q}$ corrections to $\mathcal{L}_{0}$. 
The effective Lagrangian we have constructed to describe the low energy dynamics of heavy mesons reads [1,2]:

$$
\begin{aligned}
\mathcal{L}_{\mathrm{eff}}= & \bar{h}_{v} i v \cdot \partial h_{v}+\bar{q}\left(i \not D-m_{q}\right) q-\frac{1}{4} F_{a}^{\mu \nu} F_{a \mu \nu} \\
& +P_{v}^{\dagger}(i v \cdot \stackrel{\leftrightarrow}{\partial}-2 \bar{\Lambda}) P_{v}-V_{v}^{\mu \dagger}(i v \cdot \stackrel{\leftrightarrow}{\partial}-2 \bar{\Lambda}) V_{v \mu} \\
& -\left(\bar{h}_{v} i \gamma_{5} q_{v} P_{v}-\bar{h}_{v} \gamma_{\mu} q_{v} V_{v}^{\mu}+h . c .\right) \\
& +\sum_{n=1}^{\infty}\left(\frac{1}{2 m_{Q}}\right)^{n} \bar{h}_{v}\left(i \not D_{\perp}\right)(-i v \cdot D)^{n-1}\left(i \not D_{\perp}\right) h_{v} \\
= & \mathcal{L}_{\mathrm{eff}}^{Q q H}+\mathcal{L}_{m_{Q}},
\end{aligned}
$$

where $P_{v}$ and $V_{v}^{\mu}$ represent respectively the composite pseudoscalar and vector heavy meson fields which appear only as external states,

$$
\bar{\Lambda} \equiv \lim _{m_{Q} \rightarrow \infty}\left(M_{H}-m_{Q}\right)
$$

is their common residual mass in the heavy quark limit,

$$
q_{v}=G \Psi\left(v \cdot p_{q}\right) q
$$

represents collectively the light degrees of freedom in a heavy meson, where $\Psi(v \cdot p)$ is heavy meson wave function mentioned earlier, $p_{q}$ is the light antiquark momentum, and $G$ is the normalization constant given by

$$
G^{-2}=i \int \frac{d^{4} p_{q}}{(2 \pi)^{4}} \frac{\left|\Psi\left(v \cdot p_{q}\right)\right|^{2}}{\left(\bar{\Lambda}-v \cdot p_{q}+i \epsilon\right)^{2}} \frac{v \cdot p_{q}+m_{q}}{\left(p^{2}-m_{q}^{2}+i \epsilon\right)} .
$$

In Eq.(2.3), $\mathcal{L}_{\text {eff }}^{Q q H}$ obeys HQS and defines the nonperturbative effective coupling of heavy mesons with their constituent heavy and light quarks, while $\mathcal{L}_{m_{Q}}$, which contains all the $1 / m_{Q}$ corrections, is considered as a perturbation to $\mathcal{L}_{\mathrm{eff}}^{Q q H}$. A practical calculational scheme has been developed in terms of the Feynman diagrammatic rules summarized below [1,2]:

(i) A heavy meson bound state $\left(p_{H}=\bar{\Lambda} v\right)$ couples to its constituent heavy and light quarks via a three-point vertex

$$
--\quad: \quad-i G \Psi\left(v \cdot p_{q}\right) \Gamma_{H}
$$




$$
-i G \Psi^{*}\left(v \cdot p_{q}\right) \Gamma_{H}
$$

where $\Gamma_{P}=i \gamma_{5}$ for pseudoscalar $(H=P), \Gamma_{V}=-\notin$ for vector mesons $(H=V)$, and $p_{q}$ is the momentum of the light degrees of freedom. Each vertex also carries a momentum conservation factor of $(2 \pi)^{4} \delta^{4}\left(\bar{\Lambda} v-k-p_{q}\right)$.

(ii) The internal heavy quark and light quark propagators are,

$$
\begin{array}{cc}
\bar{k} & : \quad i \frac{\not p+1}{2(v \cdot k+i \epsilon)}, \\
\frac{-p_{q}}{\tau^{2}} & : \quad i \frac{-\not p_{q}+m_{q}}{p_{q}^{2}-m_{q}^{2}+i \epsilon},
\end{array}
$$

respectively, where $k$ is the residual momentum of the heavy quark, and $m_{q}$ the constituent mass of the light quark.

(iii) An integration factor $\int d^{4} p /(2 \pi)^{4}$ is associated with each internal momentum $p$, and a factor $(-1)$ should be included for each fermion loop.

(iv) For all other lines and vertices not attached to heavy mesons (mostly from $\mathcal{L}_{m_{Q}}$ ), standard Feynman rules apply. Hence $1 / m_{Q}$ corrections to various quantities can be calculated using standard field theoretic perturbative approach [2].

\section{B. Heavy meson bound state equation}

In HQET, the reduced heavy quark field $h_{v}$ obeys the following equation of motion

$$
i v \cdot D h_{v}=0
$$

where $D^{\mu}=\partial^{\mu}-i g A^{\mu}$ is the QCD covariant derivative.

To ensure that our effective field theory, Eq.(2.3), is consistent with low energy QCD dynamics of HQET, Eq.(2.1), we require that in the heavy quark limit, the heavy meson state $|H(v)\rangle$ satisfies

$$
\left\langle H_{v}\left|\bar{h}_{v} i v \cdot D h_{v}\right| H_{v}\right\rangle=0
$$


Strickly speaking, this matrix element must be calculated in a nonperturbative manner of QCD, which is however beyond our present day knowledge of QCD. To circumvent this predicament, we shall adopt an effective gluon propagator which contains a long-distance contribution as well as the familiar perturbative one [6]:

$$
g_{s}^{2} \Pi^{\mu \nu}(q)=-i g^{\mu \nu} \delta_{a b}\left(\frac{g_{s}^{2}}{q^{2}+i \epsilon}+i 4 \pi^{2} \Lambda_{c}^{2} \delta^{4}(q)\right)
$$

where $g_{s}$ is the QCD coupling constant, and the coefficient $\Lambda_{c}^{2}$ characterizes the confining strength. Note that the regularized infrared singular term $\delta^{4}(q)$ can be effectively considered as a regularization of the linearly confining interaction $1 / q^{4}$ [6]. Representing this effective gluon propagator by a double wavy line, we can depict the left hand side of Eq. (2.12) pictorially by Feynman diagrams shown in Fig. 1.

As discussed in [1,2], from the constraint that it forbids on-mass-shell decay of the heavy meson into $Q \bar{q}$, a possible form of the heavy meson wave function $\Psi\left(v \cdot p_{q}\right)$ is given by

$$
\Psi\left(v \cdot p_{q}\right)=\left(\bar{\Lambda}-v \cdot p_{q}\right) \varphi\left(v \cdot p_{q}\right)
$$

where the function $\varphi\left(v \cdot p_{q}\right)$ is regular at $v \cdot p_{q}=\bar{\Lambda}$. Then, applying the diagrammatic rules stated in Sec. IIA to Fig. 1, we can easily translate Eq.(2.12) into the following expression,

$$
\begin{aligned}
& i G^{2} \int \frac{d^{4} p_{q}}{(2 \pi)^{4}}\left|\varphi\left(v \cdot p_{q}\right)\right|^{2} \frac{v \cdot p_{q}+m_{q}}{p^{2}-m_{q}^{2}}\left(\bar{\Lambda}-v \cdot p_{q}\right) \\
&+C_{f} G^{2} \int \frac{d^{4} p_{q}}{(2 \pi)^{4}} \frac{d^{4} p_{q}^{\prime}}{(2 \pi)^{4}} \varphi\left(v \cdot p_{q}\right) \varphi^{*}\left(v \cdot p_{q}^{\prime}\right)\left(\frac{4 \pi \alpha_{s}}{\left(p_{q}-p_{q}^{\prime}\right)^{2}}+i 4 \pi^{2} \Lambda_{c}^{2} \delta^{4}\left(p_{q}-p_{q}^{\prime}\right)\right) \\
& \quad \times \frac{\left(v \cdot p_{q}+m_{q}\right)\left(v \cdot p_{q}^{\prime}+m_{q}\right)+v \cdot p_{q} v \cdot p_{q}^{\prime}-p_{q} \cdot p_{q}^{\prime}}{\left(p_{q}^{2}-m_{q}^{2}\right)\left(p_{q}^{\prime 2}-m_{q}^{2}\right)} \\
&=0,
\end{aligned}
$$

where the color factor $C_{f}=\left(N_{c}^{2}-1\right) / 2 N_{c}=4 / 3$ for $N_{c}=3$. The above equation can be rewritten as an equivalent bound state equation for heavy mesons:

$$
\begin{aligned}
\left(\bar{\Lambda}-v \cdot p_{q}\right) \varphi\left(v \cdot p_{q}\right)= & C_{f} \int \frac{d^{4} p_{q}^{\prime}}{(2 \pi)^{4}}\left(\frac{i 4 \pi \alpha_{s}}{\left(p_{q}-p_{q}^{\prime}\right)^{2}}-4 \pi^{2} \Lambda_{c}^{2} \delta^{4}\left(p_{q}-p_{q}^{\prime}\right)\right) \\
& \times \frac{\left(v \cdot p_{q}+m_{q}\right)\left(v \cdot p_{q}^{\prime}+m_{q}\right)+v \cdot p_{q} v \cdot p_{q}^{\prime}-p_{q} \cdot p_{q}^{\prime}}{\left(v \cdot p_{q}+m_{q}\right)\left({p^{\prime}}_{q}^{2}-m_{q}^{2}\right)} \varphi\left(v \cdot p_{q}^{\prime}\right),
\end{aligned}
$$


which is indeed a covariant generalization of the light-front bound state equation for heavy mesons we have previously derived [3], and it is also the heavy quark limit of the BetheSalpeter equation for heavy mesons.

Using the normalization condition of $\Psi\left(v \cdot p_{q}\right)$, we can simplified Eq.(2.15) to

$$
\begin{gathered}
\bar{\Lambda}=i G^{2} \int \frac{d^{4} p_{q}}{(2 \pi)^{4}}\left|\varphi\left(v \cdot p_{q}\right)\right|^{2} \frac{v \cdot p_{q}+m_{q}}{p^{2}-m_{q}^{2}} v \cdot p_{q} \\
-C_{f} G^{2} \int \frac{d^{4} p_{q}}{(2 \pi)^{4}} \frac{d^{4} p_{q}^{\prime}}{(2 \pi)^{4}} \varphi\left(v \cdot p_{q}\right) \varphi^{*}\left(v \cdot p_{q}^{\prime}\right)\left(\frac{4 \pi \alpha_{s}}{\left(p_{q}-p_{q}^{\prime}\right)^{2}}+i 4 \pi^{2} \Lambda_{c}^{2} \delta^{4}\left(p_{q}-p_{q}^{\prime}\right)\right) \\
\quad \times \frac{\left(v \cdot p_{q}+m_{q}\right)\left(v \cdot p_{q}^{\prime}+m_{q}\right)+v \cdot p_{q} v \cdot p_{q}^{\prime}-p_{q} \cdot p_{q}^{\prime}}{\left(p_{q}^{2}-m_{q}^{2}\right)\left(p_{q}^{\prime 2}-m_{q}^{2}\right)} .
\end{gathered}
$$

The physical meaning of the above result is clear. The heavy meson binding energy $\bar{\Lambda}$ defined by Eq.(2.4) consists of two parts: One is the light quark kinetic energy contribution $\left\langle v \cdot p_{q}\right\rangle$, and the other is the interaction energy of the heavy-light system. Note that, due to the effective gluon propagator (2.13) used, the interaction energy contains a confining contribution, as well as the color Coulomb contribution.

\section{Variational determination of heavy meson wave function $\varphi\left(v \cdot p_{q}\right)$}

In this section we shall determine the heavy meson wave function $\varphi\left(v \cdot p_{q}\right)$ through a variational procedure. The first step is to choose an appropriate trial wave function. As discussed in [1,2], we demand that the wave function should be relatively well behaved, by which we mean that, when continued into the complex $p_{q}$ plane, (i) $\varphi\left(v \cdot p_{q}\right)$ is analytic except for isolated singularities, and (ii) it vanishes "fast enough" as $|v \cdot q| \rightarrow \infty$. These two constraints also allow us to easily derive various relativisitic and/or light-front quark models results as special cases in our effective field theory [1.2].

In various existing light-front or relativistic quark models, two types of distribution functions have been widely adopted for phenomenological hadronic wave functions. One is the exponential(Gaussian)-type and the other the Lorentzian-type. The former can be

excluded since it does not simultaneously satisfy the two constraints stated above [4:2]. On the other hand, we find that Lorentzian-type functions of the form, 


$$
\varphi_{n}\left(v \cdot p_{q}\right)=\frac{1}{\left(v \cdot p_{q}+\omega-i \epsilon\right)^{n}}
$$

do meet our requirements [1,2], and will be taken as the working trial heavy meson wave function in this work. It is interesting to note that such a choice of $\varphi\left(v \cdot p_{q}\right)$ corresponds to a covariant light-front wave function [7] of the form

$$
\varphi_{L F}^{n}\left(v \cdot p_{q}\right)=\mathcal{N}_{L} \frac{\sqrt{v \cdot p_{q}+m_{q}}}{\left(v \cdot p_{q}+\omega\right)^{n}} \quad n>4
$$

where $\mathcal{N}_{L}$ is a normalization constant, $p_{q}^{2}=m_{q}^{2}$, and $n>4$ is required to ensure convergence.

For the sake of demonstration, we first consider the naive case of ignoring of the confining interaction between the heavy quark and the light degrees of freedom, i.e. $\Lambda_{c}=0$, so that color Coulomb interaction is solely responsible for binding the quarks together. We find that, for $n=6$, a minimum with $\bar{\Lambda}=0.192 \mathrm{GeV}$ can be found at $\omega=0.03$. However a wave function with such a small $\omega$ is unrealistic because it contains too little high momentum components. In other words, the wave function is too extended in configuration space, as would be expected since we have ignored the confining part of the inter-quark interaction.

With an appropriate non-zero $\Lambda_{c}$, physically sensible results can be obtained. In what follows, we will take $\alpha_{s}=0.44$ and $m_{q}=0.22 \mathrm{GeV}$, which are close to the values used in our previous work [2]. With the confinement strength taken to be $\Lambda_{c}=0.665 \mathrm{GeV}$, we determine the wave function parameter $\omega$ by minimizing $\bar{\Lambda}$. The results are listed in the Table I, where

$$
\bar{\Lambda}=E_{\mathrm{ke}}+E_{\mathrm{conf}}+E_{\mathrm{coul}}=0.426 \sim 0.430 \mathrm{GeV}
$$

which is not sensitive to the choice of $n$. This value of $\bar{\Lambda}$ is somewhat larger than our previous result of $\bar{\Lambda}=0.33 \sim 0.34 \mathrm{GeV}$ obtained in [2]. The difference is mainly due to the confinement effect which is not considered there. 
Table I. The wave function parameter $\omega$ nonperturbatively determined from bound state equation.

\begin{tabular}{|c|c|c|c|c|c|}
\hline \hline$\phi_{n}\left(v \cdot p_{q}\right)$ & $\omega$ & $\left\langle v \cdot p_{1}\right\rangle$ & $E_{\text {conf }}$ & $E_{\text {coul }}$ & $\bar{\Lambda}$ \\
\hline$n=6$ & 0.80 & 0.443 & 0.147 & -0.160 & 0.430 \\
\hline$n=7$ & 1.01 & 0.440 & 0.147 & -0.158 & 0.427 \\
\hline$n=8$ & 1.24 & 0.438 & 0.144 & -0.157 & 0.426 \\
\hline \hline
\end{tabular}

It is worth emphasizing that, in our previous work [2], the parameter $\omega$ is fixed phenomenologically by fitting to $f_{B}=180 \mathrm{MeV}$ from lattice gauge calculations (it is experimentally unknown). In the present work, we have dynamically determine the wave function $\varphi(v \cdot p)$ by minimizing the blinding energy $\bar{\Lambda}$ from the QCD-based bound state equation. This is a significant improvement which helps to raise the predictive power of our heavy meson effective theory.

\section{PSEUDSCALAR AND VECTOR HEAVY MESON MASSES}

In this section, we shall use the bound state solution obtained in the previous section to calculate heavy meson masses up to order $1 / m_{Q}$. This will in turn yield more consistent results for $\lambda_{1}$ and $\lambda_{2}$ than those obtained in [2].

In the heavy quark limit, the heavy meson masses can be written as $M_{H}=m_{Q}+\bar{\Lambda}$. The correction to $M_{H}$ in HQET comes mainly from the leading HQS-breaking $1 / m_{Q}$ corrections [see Eq. (2.3)]:

$$
\mathcal{L}_{1}=\bar{h}_{v}\left(i D_{\perp}\right)^{2} h_{v}+\frac{g_{s}}{2} \bar{h}_{v} \sigma_{\mu \nu} G^{\mu \nu} h_{v}=\mathcal{O}_{1}+\mathcal{O}_{2}
$$

where $\sigma_{\mu \nu}=\frac{i}{2}\left[\gamma_{\mu}, \gamma_{\nu}\right]$ and $G^{\mu \nu}=\frac{i}{g_{s}}\left[D^{\mu}, D^{\nu}\right]$. With these $1 / m_{Q}$ corrections included, the heavy meson masses can be expressed as

$$
M_{H}=m_{Q}+\bar{\Lambda}-\frac{1}{2 m_{Q}}\left(\lambda_{1}+d_{H} \lambda_{2}\right),
$$

where $\lambda_{1}$ comes from $\mathcal{O}_{1}, d_{H} \lambda_{2}$ from $\mathcal{O}_{2}$ (see Fig. 3), and $d_{H}=3(-1)$ for pseudoscalar (vector) mesons [2]. In Eq. (3.2), $\lambda_{1}$ parametrizes the common mass shift, and $\lambda_{2}$ accounts 
for the so called hyperfine mass splitting between the vector and pseudoscalar heavy meson. They can be directly calculated once the heavy meson wave function $\varphi(v \cdot p)$ is determined from the variational approach presented in Sec. IIC.

Expressions for $\lambda_{1}$ and $\lambda_{2}$ are essentially the same as those given in [2], except that here we use the effective gluon propagator (2.13), which is more appropriate for physics in the low energy regime. Thus we have

$$
\begin{aligned}
\lambda_{1}=i 2 G^{2} \int \frac{d^{4} p_{q}}{(2 \pi)^{4}}\left|\varphi\left(v \cdot p_{q}\right)\right|^{2} \frac{v \cdot p_{q}+m_{q}}{p_{q}^{2}-m_{q}^{2}}\left[p_{q}^{2}-\left(v \cdot p_{q}\right)^{2}\right] \\
-2 C_{f} G^{2} \int \frac{d^{4} p_{q}}{(2 \pi)^{4}} \frac{d^{4} p_{q}^{\prime}}{(2 \pi)^{4}} \varphi\left(v \cdot p_{q}\right) \varphi^{*}\left(v \cdot p_{q}^{\prime}\right) \frac{1}{\left(p_{q}^{2}-m_{q}^{2}\right)\left(p^{\prime 2}-m_{q}^{2}\right)} \\
\times\left(\frac{4 \pi \alpha_{s}}{\left(p_{q}-p_{q}^{\prime}\right)^{2}}+i 4 \pi^{2} \Lambda_{c}^{2} \delta^{4}\left(p_{q}-p_{q}^{\prime}\right)\right) \\
\times\left\{\left(v \cdot p_{q}+m_{q}\right)\left[\left(p_{q}+p_{q}^{\prime}\right) \cdot p_{q}^{\prime}-v \cdot\left(p_{q}+p_{q}^{\prime}\right) v \cdot p_{q}^{\prime}\right]\right. \\
\left.\quad+\left(v \cdot p_{q}^{\prime}+m_{q}\right)\left[\left(p_{q}+p_{q}^{\prime}\right) \cdot p_{q}-v \cdot\left(p_{q}+p_{q}^{\prime}\right) v \cdot p_{q}\right]\right\}
\end{aligned}
$$

and

$$
\begin{aligned}
\lambda_{2}=\frac{4}{3} C_{f} G^{2} \int \frac{d^{4} p_{q}}{(2 \pi)^{4}} & \frac{d^{4} p_{q}^{\prime}}{(2 \pi)^{4}} \varphi\left(v \cdot p_{q}\right) \varphi^{*}\left(v \cdot p_{q}^{\prime}\right) \frac{1}{\left(p_{q}^{2}-m_{q}^{2}\right)\left(p_{q}^{\prime 2}-m_{q}^{2}\right)} \\
\times & \left(\frac{4 \pi \alpha_{s}}{\left(p_{q}-p_{q}^{\prime}\right)^{2}}+i 4 \pi^{2} \Lambda_{c}^{2} \delta^{4}\left(p_{q}-p_{q}^{\prime}\right)\right) \\
\times & \left\{\left(v \cdot p_{q}+m_{q}\right)\left[\left(p_{q}-p_{q}^{\prime}\right) \cdot p_{q}^{\prime}-v \cdot\left(p_{q}-p_{q}^{\prime}\right) v \cdot p_{q}^{\prime}\right]\right. \\
& \left.-\left(v \cdot p_{q}^{\prime}+m_{q}\right)\left[\left(p_{q}^{\prime}-p_{q}^{\prime}\right) \cdot p_{q}-v \cdot\left(p_{q}-p_{q}^{\prime}\right) v \cdot p_{q}\right]\right\} .
\end{aligned}
$$

Note that since the chromo-magnetic interaction vanishes at $q=0\left(p_{q}=p_{q}^{\prime}\right), \lambda_{2}$ is actually not affected by the confinement part of the gluon propagator. Now, we can use a self consistent method to determine uniquely the parameters of our effective theory $\left(\Lambda_{c}, \omega\right)$, and also those of $\operatorname{HQET}\left(\bar{\Lambda}, \lambda_{1}, \lambda_{2}\right.$, and $\left.m_{Q}\right)$. The procedure goes as follows. For a given $\Lambda_{c}$, we first determine the wave function parameter $\omega$ by minimizing the binding energy $\bar{\Lambda}$ via Eq.(2.17). Then we can evaluate $\lambda_{1}$ and $\lambda_{2}$ using Eqs.(3.3) and (3.4). From Eq. (3.2), the hyperfine mass splitting is given by

$$
\Delta M_{V P}=M_{V}-M_{P}=\frac{2 \lambda_{2}}{m_{Q}},
$$


which, together with the experimental data $\Delta M_{B^{*} B}=45.7 \pm 0.4 \mathrm{MeV}$ and $\Delta M_{D^{*} D}=$ $142.12 \pm 0.07 \mathrm{MeV}$ [10], uniquely determines the heavy quark masses $m_{b}$ and $m_{c}$. Finally, knowing $M_{B}=5.279 \mathrm{GeV}$, we can recalculate $\bar{\Lambda}$ from Eq.(3.2). This resulting $\bar{\Lambda}$ should be equal to that determined earlier from variation. If not, the process is repeated with a new $\Lambda_{c}$ until we reach consistency. Through this self consistent procedure, we can for the first time determine uniquely all the basic HQET parameters $m_{b}, m_{c}, \bar{\Lambda}, \lambda_{1}$ and $\lambda_{2}$ from QCD-based dynamics in the low energy regime. The final results are listed in the Table II.

Table II. The wave function parameter $\omega$ is variationally determined by minimizing $\bar{\Lambda}$, and all the other HQET parameters are calculated self consistently in the heavy meson effective theory with $m_{q}=0.22 \mathrm{GeV}$ and $\alpha_{s}=0.44 . \Lambda_{c}, \omega, m_{b}, m_{c}$, and $\bar{\Lambda}$ are in $\mathrm{GeV}$, and $\lambda_{1}$ and $\lambda_{2}$ in $\mathrm{GeV}^{2}$.

\begin{tabular}{|c|c|c|c|c|c|c|c|c|}
\hline \hline$\Psi_{n}\left(v \cdot p_{q}\right)$ & $\Lambda_{c}$ & $\omega$ & $\bar{\Lambda}$ & $-\lambda_{1}$ & $\lambda_{2}$ & $\Delta M_{B^{*} B}$ & $m_{b}$ & $m_{c}$ \\
\hline$n=6$ & 0.665 & 0.805 & 0.430 & 0.228 & 0.1116 & 0.0459 & 4.860 & 1.570 \\
\hline$n=7$ & 0.685 & 1.070 & 0.436 & 0.247 & 0.1114 & 0.0459 & 4.852 & 1.568 \\
\hline$n=8$ & 0.705 & 1.336 & 0.443 & 0.264 & 0.1112 & 0.0459 & 4.845 & 1.556 \\
\hline exp. & - & - & $0.39 \pm 0.11[1]$ & $0.19 \pm 0.1[11]$ & $0.1117^{*}$ & $0.0457 \pm 0.004$ & $4.89 \pm 0.05^{* *}$ & $1.59 \pm 0.02^{* *}$ \\
\hline \hline
\end{tabular}

*In the literature, the so-called "experimental" value $\lambda_{2} \simeq 0.12 \mathrm{GeV}^{2}$ is obtained by assuming $m_{b}=M_{B}$. One would get $\lambda_{2} \simeq 0.1117 \mathrm{GeV}^{2}$ if the pole-mass $m_{b}^{\text {pole }}=4.89 \mathrm{GeV}$ is used in Eq. (3.5) for $\Delta M_{B^{*} B}=45.7$ $\mathrm{MeV}$.

** There are no experimental data for the constituent quark masses $m_{b}$ and $m_{c}$, and the pole masses are displaced [12].

\section{SUMMARY AND CONCLUSION}

We know that HQS and confinement mechanism are crucial ingredients of nonperturbative QCD dynamics in heavy hadrons. However they are rarely incorporated in quark models in a fully covariant manner. In this work, we attempt to establish a link between quark model and low-energy QCD dynamics in an effective field theoretic approach, in which 
the heavy meson wave function is determined variationally. Our effective theory preserves the simplicity of a conventional quark model; in fact, for heavy quark transitions, we can reproduce the covariant light-front quark model [7] as a special case. The light-front quark model has been widely used to describe heavy meson structures. However, because its relation to QCD is not clear, it is incapable of describing the hadronic mass spectrum. Moreover, the model is not fully covariant, so that it can only describe physical processes with spacelike momentum transfers [8,9]. In the present formalism, we can study hadronic bound state physics in a covariant framework; moreover, a connection to low-energy QCD dynamics has also been established. Thus our heavy meson effective theory, together with the nonperturbatively determinated heavy meson wave function, provides a useful QCD based framework in which to study heavy quark physics in the low energy regime.

We have adopted a self-consistent procedure to determine the heavy meson wave function, the binding energy $\bar{\Lambda}$, as well as its $1 / m_{Q}$ corrections $\lambda_{1}$ and $\lambda_{2}$. ¿From these HQET parameters, we can determine the heavy quark masses $m_{b}$ and $m_{c}$ in the low energy regime by fitting to the $B^{*}-B$ and $D^{*}-D$ hyperfine mass differences. For these static properties, our results are consistent with other studies on inclusive B-meson decays [ [⿴囗十].

A great advantage of our formalism is that we can unambiguously study hadronic transition processes at arbitrary momentum transfers. Using the heavy meson wave function determined in this paper, we can further calculate dynamically the heavy meson decay constant (which is usually considered as an input in various quark models for heavy mesons) and other hadronic properties such as the Isgur-wise function, $D D^{*} \pi$ and $D D^{*} \gamma$ coupling constants, and also their $1 / m_{Q}$ corrections. The results will be presented in forthcoming papers.

\section{ACKNOWLEDGMENTS}

This work is supported in part by National Cheng-Kung University, and National Science Council of the Republic of China under Grants NSC87-2112-M-001-002, NSC88-2112-M-001- 
012. 


\section{REFERENCES}

[1] C. Y. Cheung and W. M. Zhang, Mod. Phys. Lett. A13, 2163 (1998).

[2] H. Y. Cheng, C. Y. Cheung, and W. M. Zhang, Phys. Rev. D58, 74003 (1998).

[3] W. M. Zhang, Phys. Rev. D56, 1528 (1997).

[4] M. Gremm et al. Phys. Rev. Lett. 77, 20 (1996); V. Chernyak, Phys. Lett. B387, 173 (1996); A. F. Fack, M. Like and M. J. Savage, Phys. Rev. D53, 6316 (1996); M. Gremm and I. Stewart, idib, D55, 1226 (1997); H. Li and H.-L. Yu, idib, D55, 2833 (1997).

[5] H. Georgi, Phys. Lett. B240, 447 (1990); E. Eichten and B. Hill, Phys. Lett. B234, 511 (1990); B243, 427 (1990); also see review article by M. Neubert, Phys. Rep. 245, $261(1994)$.

[6] S. Mandelstam, Phys. Rev. D20, 3223 (1979); U. Bar-Gadda, Nucl. Phys. B163, 312 (1980); H. Munczek and A. Nemirocsky, Phys. Rev. D28, 181 (1983)

[7] H. Y. Cheng, C. Y. Cheung, C. W. Hwang, and W. M. Zhang, Phys. Rev. D57, 5598 (1998).

[8] C. Y. Cheung, C. W. Hwang and W. M. Zhang, Z. Phys. C75, 657 (1997).

[9] H. Y. Cheng, C. Y. Cheung, and C. W. Hwang, Phys. Rev. D55, 1559 (1997).

[10] Particle Data Group, Phys. Rev. D54, 1 (1996).

[11] M. Gremm et al., in Ref. [4]

[12] H. Fusaoka and Y. Koide, Phys. Rev. D57, 3886 (1998). 


\section{Figure Captions}

Fig. 1 Diagram for heavy meson bound states: (a) kinetic energy contribution and (b) effective one-gluon exchange contribution which contains the Columbus interaction plus confining interaction in heavy quark symmetry. 


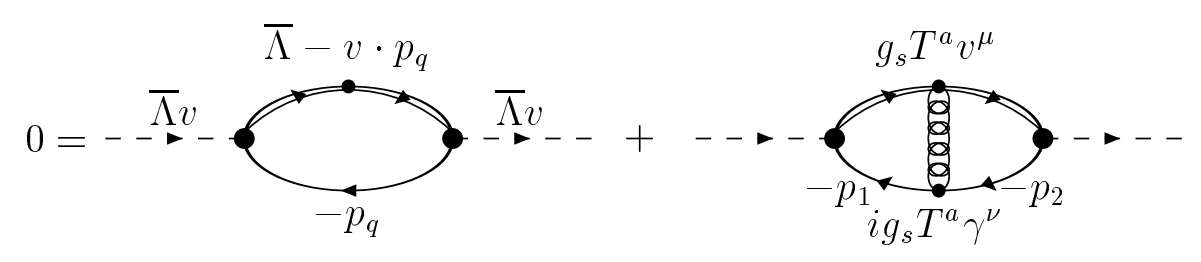

